\title{
CONGENITAL LOBAR EMPHYSEMA
}

\author{
BY \\ A. HOLZEL, E. BENNETT, and B. F. VAUGHAN \\ From the Department of Child Health, the University of Manchester, and Booth Hall Children's Hospital, \\ Manchester
}

(RECEIVED FOR PUBLICATION FEBRUARY 21, 1956)

Congenital lobar emphysema, though an established clinical entity, seems a relatively rare condition if judged by the small number of cases reported, particularly in this country (White-Jones and Temple, 1954; Belcher and Siddons, 1954). It is, however, likely that a more widespread knowledge of the clinical manifestations will reveal it as a not altogether uncommon disease of the very young.

Generally the affected infants present the picture of an acute respiratory emergency in the neonatal period or during the first few months of life. There is usually a history of recurrent attacks of dyspnoea and cyanosis which tend to increase in severity. Wheezing is often a marked feature of the condition, as well as recession of the intercostal spaces and indrawing of the suprasternal area. Examination suggests emphysema of one lung and radiological investigation shows the increased air content of one lobe, almost always an upper one, which herniates through the anterior mediastinum towards the opposite side. Early recognition and lobectomy may lead to complete recovery.

During the past five years three cases came under observation, each of them illustrating a particular and interesting facet of the problem and so warrant their recording.

\section{Case Reports}

Case 1. D.O., a boy, aged 4 months, was admitted to hospital on September 20, 1950, suffering from acute gastro-enteritis. There was a history of rapid respiration since birth with prominent movement of the alae nasi. Lying on the right side increased the dyspnoea and bulging of the anterior chest wall had also been noticed by his mother. On clinical examination there was indrawing of the suprasternal notch and lower ribs. There was no cyanosis. The chest was bulging centrally and there was hyperresonance with diminished air entry on the left side.

A chest radiograph (Fig. 1) showed extensive emphysema of the left upper lobe with marked mediastinal shift to the right. Compression had caused atelectasis of the left lower lobe and there was anterior mediastinal herniation of the emphysematous lobe.

As the baby's condition did not show any deterioration he was discharged from hospital and followed up as an out-patient. Clinical and radiological examination on January 19, 1951, revealed no change, but when the baby was seen again on August 28, 1951, although the clinical condition remained unaltered, radiological examination showed a definite decrease in the emphysema of the left upper lobe. He remained well and attended for further radiographs (Fig. 2) on February 29. 1952, which showed considerable improvement. The left upper lobe remained emphysematous but there was considerable reduction to the mediastinal shift and some re-expansion of the left lower lobe.

The patient re-attended on February 4, 1955, aged 5 years, for reassessment before beginning school. He appeared well developed for his age but the upper ribs were prominent with a depressed sternum. Physical examination of the chest showed hyperresonance of the

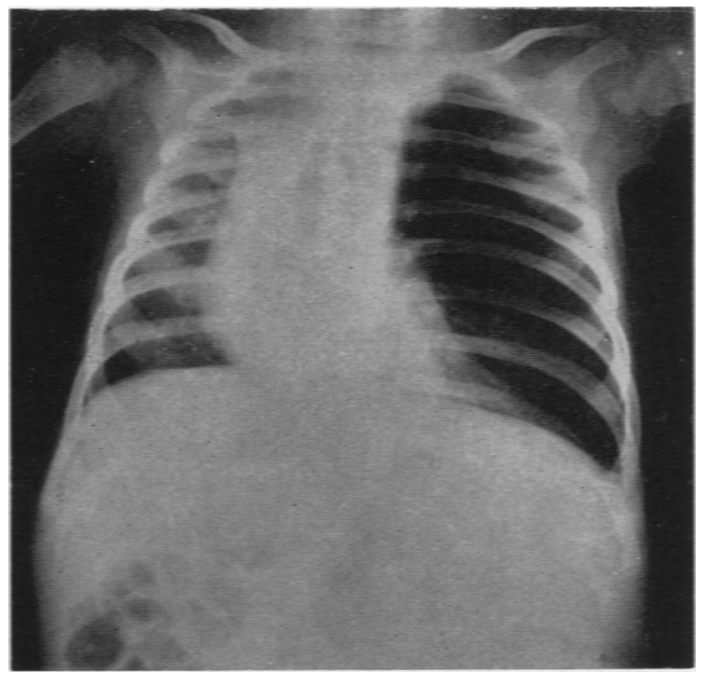

Fig. 1.-Case 1. Chest radiograph September 20, 1950, showing extensive emphysema of the left upper lobe with herniation to the right, marked mediastinal shift and compression atelectasis of the left lower lobe. 


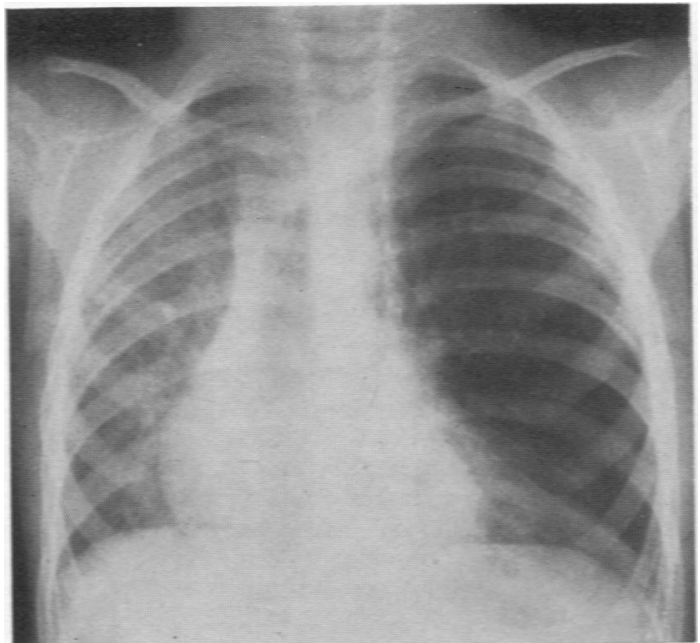

Fig. 2.-Case 1. Radiograph of the chest taken on February 29, 1952, showing reduction in the mediastinal shift and some re-expansion of the left lower lobe. The left upper lobe remains emphysematous.

left side with slightly diminished air entry. A few dry adventitious sounds were audible on either side of the sternum. His mother stated that his exercise tolerance had gradually improved and that he could walk and run quite easily without undue breathlessness. Two years previously he had had a severe attack of bronchitis which lasted eight months.

A radiograph of the chest (Fig. 3) showed only a small degree of emphysema of the left upper lobe and slight mediastinal shift to the right.

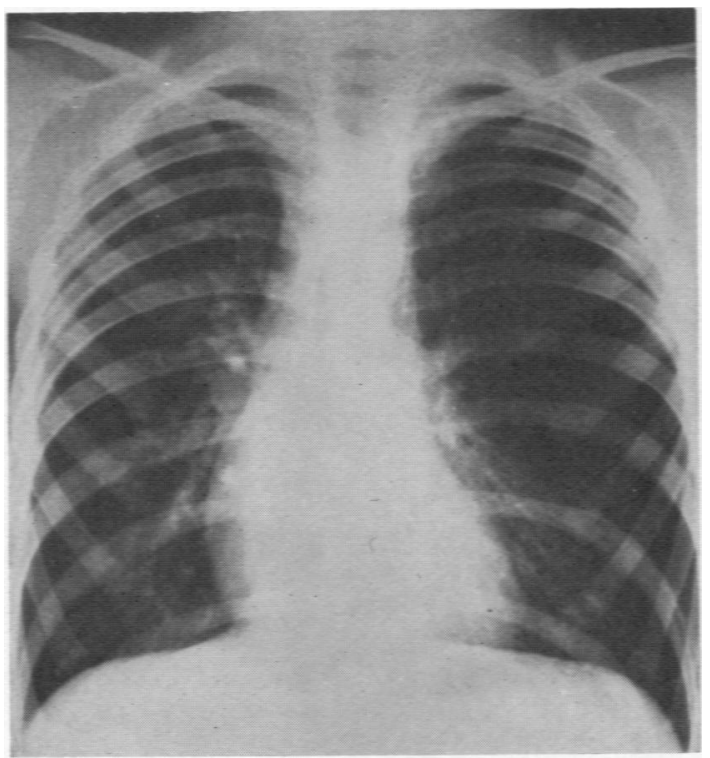

Fig. 3.-Case 1. Chest radiograph February 4, 1955, showing some emphysema still and a slight degree of mediastinal shift.
Case 2. Marguerite B. was born on June 14, 1953, at full term with a normal delivery at a large maternity hospital (birth weight $6 \mathrm{lb}$. $15 \mathrm{oz}$.). She was breast fed for eight days, then because of failing lactation put on a formula of half-cream National dried milk. Shortly after discharge from hospital her mother noticed that the baby was wheezy and short of breath. At the age of 4 weeks she had had several attacks of dyspnoea accompanied by what was thought to be colicky abdominal pain lasting about half an hour on each occasion.

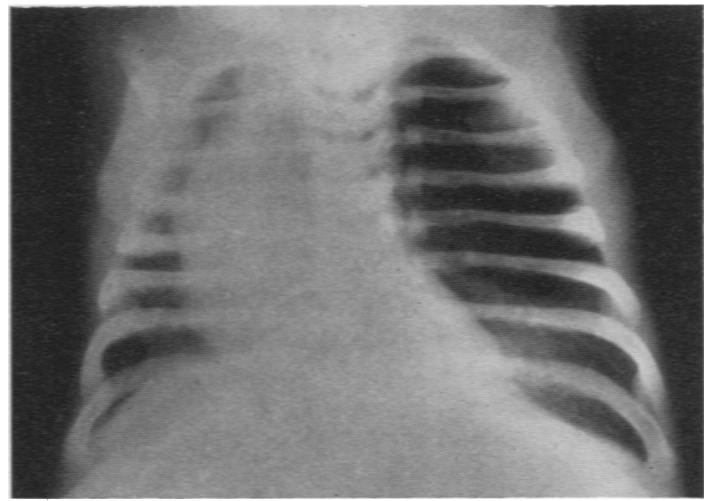

Fig. 4.- Case 2. Emphysema of the left upper lobe with herniation to the right. Considerable mediastinal shift to the right, atelectasis of the left lower lobe.

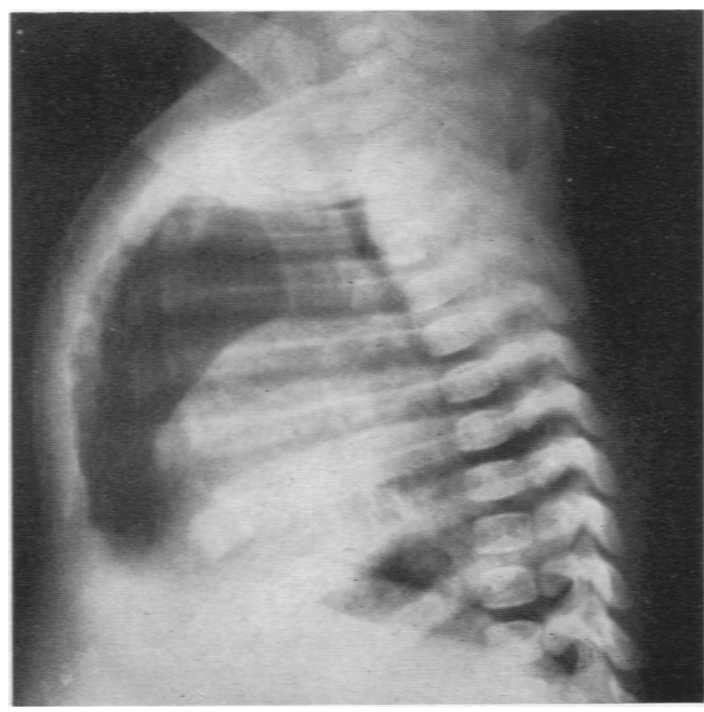

Fig. 5.-Case 2. Left lateral view, showing separation of the heart from the bulging anterior chest wall by the emphysematous lobe.

On July 30, 1953, she had several convulsions with twitching movements in the muscles of the face and left arm. Because of the persistence of the convulsions she was admitted to hospital on the same day.

The grandfather had asthma and the mother had 


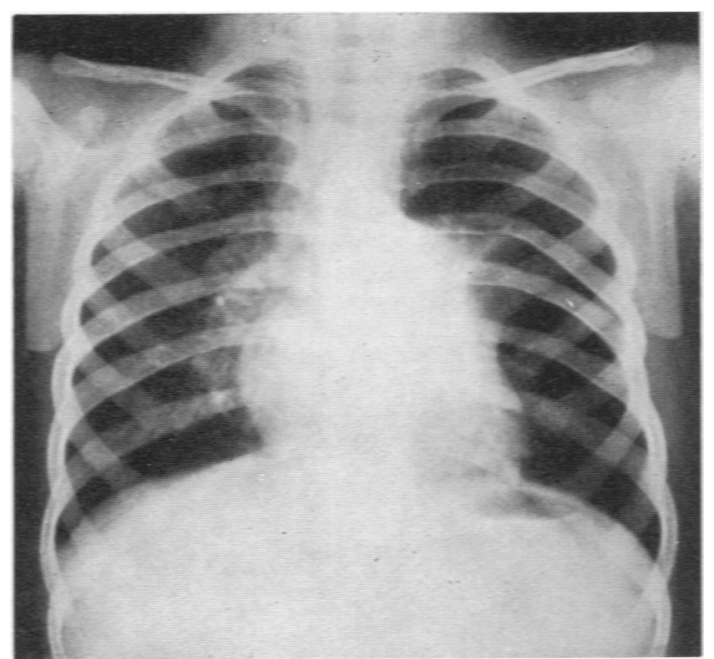

FIG. 6.-Case 2. Radiograph of chest 20 months after operation.

slight dermatitis of both hands, recurring every winter. She was suspected of having hyperthyroidism during the pregnancy.

Examination on admission showed an extremely pale, ill baby, cyanosed, very dyspnoeic and restless. There was marked wheezing, rib recession and indrawing of the suprasternal space. The percussion note over the left side of the chest was hyperresonant with greatly diminished air entry on the same side. The mediastinum was displaced to the right and the heart sounds could be heard more clearly on the right. The anterior chest wall appeared raised so that the chin practically rested on it.

A clinical diagnosis of tension pneumothorax was made and drainage under water immediately instituted, as the child's desperate condition did not allow radiological examination. Some improvement followed but no alteration in the mediastinal displacement. The thoracocentesis was stopped because of the development of subcutaneous emphysema.

Radiological examination showed an extensive obstructive emphysema of the left upper lobe with herniation to the right side through the upper part of the anterior mediastinum (Figs. 4 and 5). The baby's condition remained relatively stable, though she was still dyspnoeic, until August 14, 1953, when she suddenly collapsed, became extremely cyanosed and lost consciousness, but recovered again within 30 minutes. Because of repetition of the attacks she was bronchoscoped. Except for the deviation of the trachea no abnormality was detected.

On August 25, 1953, an exploratory thoracotomy was carried out on the left side (Mr. Dewsbury). The left upper lobe appeared grossly distended and uniformly emphysematous. The lower lobe was atelectatic and filled only a small space at the bottom of the chest cavity. The left upper lobe was resected easily. Postoperative convalescence was uneventful and she was discharged home on September 16, 1953.
Microscopical examination of the resected lobe showed only pathological changes compatible with the emphysema. The bronchus appeared normal.

The patient remained well until October 15, 1953, when she was re-admitted with signs of respiratory distress, cyanosis and marked wheezing. There were signs of upper respiratory tract infection. Despite antibiotic therapy her condition deteriorated and for the next six days she remained desperately ill; only subcutaneous injections of adrenaline relieved the respiratory distress. Following a course of cortisone she gradually recovered and was discharged home.

She was last seen in April, 1955, aged 23 months. Her general condition was excellent; she was lively, active, running about singing and had learnt to talk. A slight wheeze was audible on auscultation. In the intervening period she had had a number of attacks of varying degree of dyspnoea, always associated with wheezing and usually precipitated by a respiratory infection (Fig. 6).

Case 3. David A. was admitted to hospital on December 26, 1954, aged 11 weeks. The history was that he had been 'wheezy' since birth. Three days before admission he had developed a cough and had been noticed to become increasingly breathless.

On admission he was extremely dyspnoeic and slightly cyanosed. The anterior chest wall was raised to the level of the chin, and the chest was fixed in almost complete inspiration, respiration being mainly diaphragmatic. On auscultation there were rhonchi and fine crepitations throughout both lungs, and air entry over the left lung was markedly decreased. Radiographs of the chest on December 28, 1954 (Fig. 7) showed gross emphysema of the left upper lobe, with herniation across to the right and compression atelectasis of the left lower lobe.

He was treated with antibiotics and oxygen, and clinically his condition improved, but examination of the chest still showed hyperresonance and diminished

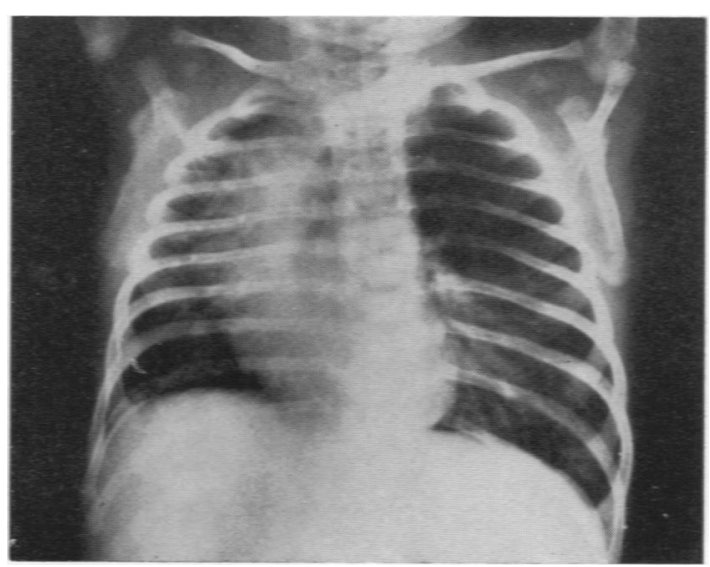

Fig. 7.-Case 3. Gross emphysema of the left upper lobe, herniation to the right, and atelectasis of the left lower lobe. 


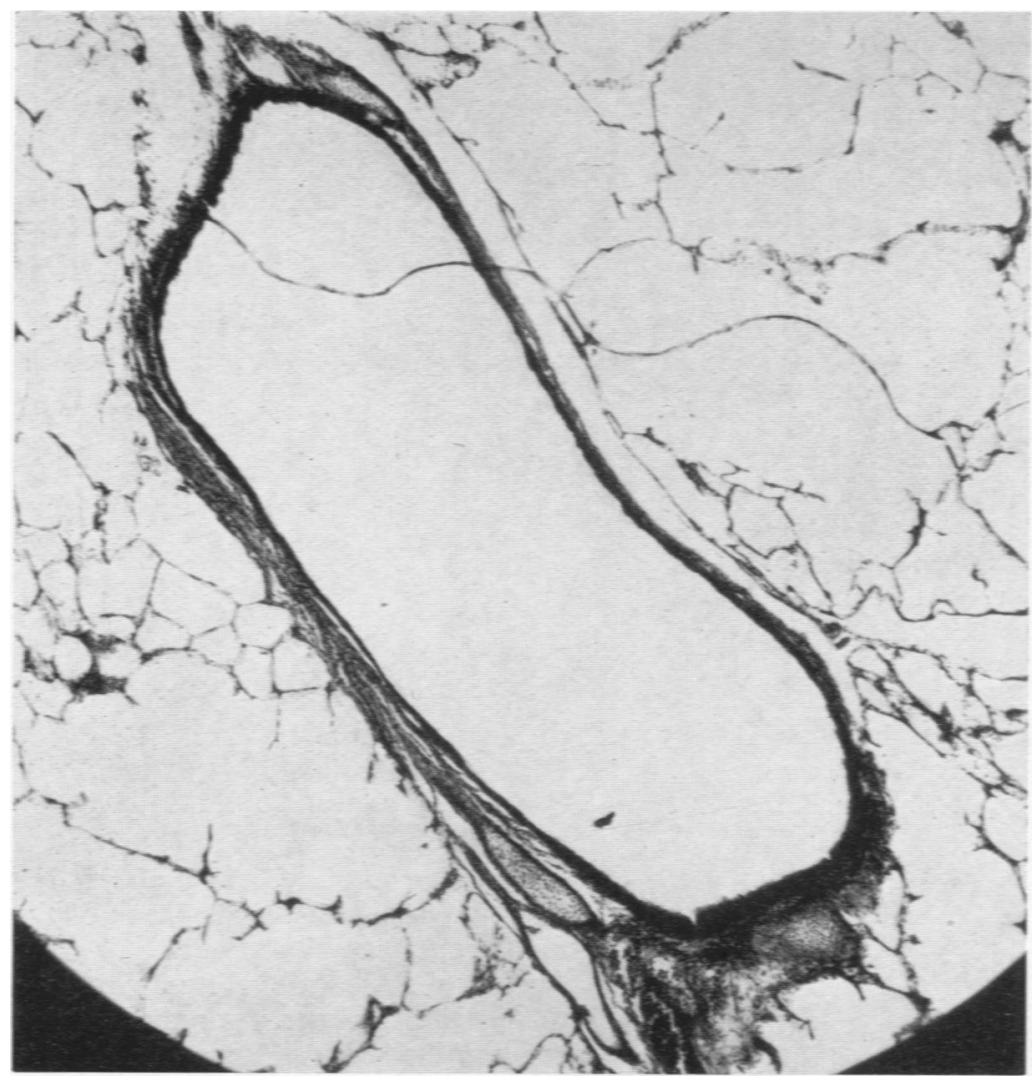

Fig. 8.-Case 3. Section of the left upper lobe showing only a very small amount of cartilage supporting a large bronchus and emphysematous changes in the parenchyma. $\times 50$.

five-year observation period the clinical signs have regressed and the patient has developed normally. He successfully overcame a severe respiratory illness. Now his exercise tolerance is normal and the only residual physical signs are bulging of the chest, with some hyperresonance, and depression of the sternum (Fig. 10).

Two similar cases of spontaneous regression of lobar emphysema are reported by Caffey (1953). Both cases were diagnosed when 5 weeks old, and were observed for two years and three and a half years respectively. The right lung was affected in one, and the left lower lobe in the other. The latest chest radiographs showed only increased translucency of the affected lobes. The ultimate fate of these cases is not yet known but it is interesting to conjecture that unrecognized this disease may be the cause of 'unequal translucency of the lungs' presenting in adults, as described by Macleod (1954). Samson

air entry throughout the left lung. Chest radiographs on January 3, 1955, and January 18, 1955, showed no change.

On February 14, 1955, left upper lobectomy was performed. At operation the left upper lobe was distended and had an unnatural spongy texture, but it was not entirely incompressible. The apical and dorsal segments were affected more severely than the lingular and anterior ones. The lower lobe was collapsed. There was nothing unusual about the lobectomy except that the upper lobe bronchus arose from the left main bronchus in two parts, one going to the apical and dorsal segments, and one to the anterior and lingular segments.

A section showed chronic emphysema. No cartilage was seen in the medium-sized bronchi examined, and only very small patches of cartilage tissue in different parts of the large bronchi (Figs. 8 and 9).

Post-operatively the child made an uninterrupted recovery.

\section{Discussion}

Our first case, although presenting the classical clinical and radiological picture, was not acutely distressed. No operation was performed. During the
(1953) mentioned the case of a young man, aged 21 years, who was examined before an operation for pectus excavatum. The $x$-ray film of the lung showed poor markings and increased translucency on the right side. At thoracotomy the surgeon found an enormously distended emphysematous middle lobe filling practically the whole of the right chest cavity. A congenital origin was presumed. It seems therefore that the condition, contrary to general opinion, is compatible with survival without surgical intervention and may run a self-limited course.

Case 2 presented at the age of 6 weeks with convulsions indicating that the condition may lead to such a degree of anoxia as to cause loss of consciousness and convulsions which subsided when it was relieved by oxygen. Post-operatively she had an extremely stormy passage and although her condition appeared subsequently satisfactory, so far as her general development was concerned, she was never free of physical signs and wheezing has been a 
marked feature from time to time. A few weeks after operation emphysema developed in the lower lobe on the same side. No definite anatomical lesions had been diagnosed to account for the development of emphysema in the upper lobe but as the condition spread post-operatively one may assume that there was some weakness of the cartilage of other bronchi on the same side.

Recurrence of respiratory symptoms postoperatively in all his patients was reported by Sloan (1953). Two of the four infants showed radiological evidence of emphysema in other portions of the lung following the lobectomy similar to this case. The explanation put forward was that the bronchial abnormality was not limited to the removed lobe. Interesting also is the fact that in one of the babies excellent temporary relief was obtained with subcutaneous injections of adrenaline, in the absence of any history of allergy in the child or his family, an experience not unlike our own.

Among reported cases absence or hypoplasia of bronchial cartilage has been described by Shaw (1952) in two cases, and by Fischer, Potts and Holinger (1952) in one out of six cases. In Case 3

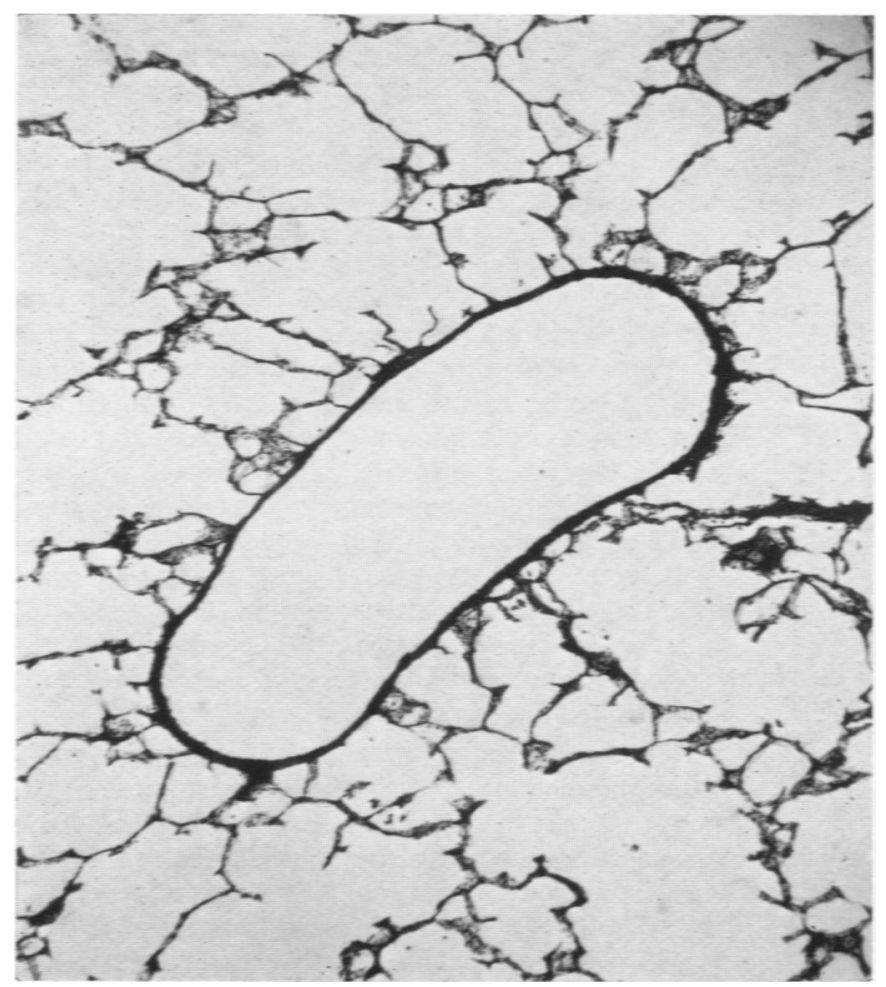

FiG. 9.-Case 3. Complete absence of cartilage in the wall of the medium-sized bronchus. $\times 50$.

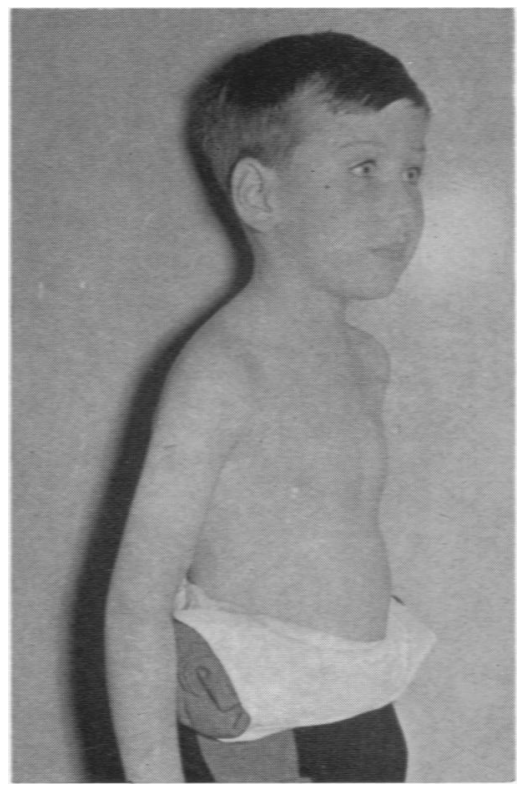

Fig. 10.-Case 1. At the age of 5 years. He has a slight chest deformity.

a similar anatomical lesion was found which explained the development of the emphysema.

In all of these cases the affected lobe has been the left upper, but in the available literature the lesion has been noted also in the right upper lobe and the right middle lobe, but only once, by Caffey (1953), in the left lower lobe. The upper lobe bronchi are generally more liable to present anatomical variations.

Despite the somewhat varied clinical manifestations of this disease the radiological appearances in all these cases were similar. These are (1) emphysema of an upper lobe; (2) collapse of a lower lobe on the ipsilateral side; (3) considerable mediastinal shift away from the lesion and anterior mediastinal herniation.

\section{Summary}

Three cases of congenital lobar emphysema are reported. In the first of them the disease was discovered accidentally at the age of 4 months. As a 5-yearold he appeared in good health without having been submitted to operation. Case 2 had severe manifestations of anoxia leading to convulsions pre- 
operatively and persistence of some degree of respiratory disability after lobectomy. Progress in the third case was uninterruptedly favourable since operation. The bronchial ramifications of the removed lobe were almost completely devoid of cartilage.

We wish to express our gratitude to Mr. Graham Bryce for permission to follow up Case 1; to Mr. Dewsbury and Mr. Dark for their cooperation and reports on the findings at operation in Cases 2 and 3; to Dr. Duran-Jorda for the histological investigation and to the Department of Medical Illustration for the $x$-ray prints. We are also indebted to Professor Wilfrid Gaisford for his kind and helpful criticism.

\section{REFERENCES}

Belcher, J. R. and Siddons, A. H. M. (1954). Thorax, 9, 38.

Caffey, J. (1953). Pediatrics, 11, 48.

Fischer, H. W., Potts, W. J. and Holinger, P. H. (1952). J. Pediat., 41, 403.

Macleod, W. M. (1954). Thorax, 9, 147.

Samson, P. C. (1953). J. thorac. Surg., $26,16$.

Shaw, R. R. (1952). Pediatrics, 9, 220.

Sloan, H. (1953). J. thorac. Surg., 26, 1.

White-Jones, R. H. and Temple, L. J. (1954). Archives of Disease in Childhood, 29, 248. 